\title{
Numerical simulations of the solar corona and Coronal Mass Ejections
}

\author{
Stefaan Poedts, Carla Jacobs, Bart van der Holst, Emmanuel Chané, and Rony Keppens \\ Centrum voor Plasma-Astrofysica, Katholieke Universiteit Leuven, Belgium
}

(Received October 31, 2007; Revised January 30, 2008; Accepted August 24, 2008; Online published May 29, 2009)

\begin{abstract}
Numerical simulations of Coronal Mass Ejections (CMEs) can provide a deeper insight in the structure and propagation of these impressive solar events. In this work, we present our latest results of numerical simulations of the initial evolution of a fast CME. For this purpose, the equations of ideal MagnetoHydroDynamics (MHD) have been solved on a three-dimensional (3D) mesh by means of an explicit, finite volume solver, where the simulation domain ranges from the lower solar corona up to $30 R_{\odot}$. In order to simulate the propagation of a CME throughout the heliosphere, a magnetic flux rope is superposed on top of a stationary background solar (MHD) wind with extra density added to the flux rope. The flux rope is launched by giving it an extra initial velocity in order to get a fast CME forming a 3D shock wave. The magnetic field inside the initial flux rope is described in terms of Bessel functions and possesses a high amount of twist.
\end{abstract}

Key words: Magnetohydrodynamics, numerical, coronal mass ejections.

\section{Introduction}

It is generally accepted that coronal mass ejections (CMEs) originate from the so-called 'closed' magnetic regions on the Sun, consisting of thousands of magnetic loops. Such 'closed' magnetic fields can be found in active regions, filaments, and transequatorial interconnection regions. The latter regions are most likely to appear during solar minimum, when the active regions are located, on average, much closer to the equator. Cremades and Bothmer (2004) studied 124 structured CME events and analysed the relation between the source region characteristics at the solar surface and the morphology of the corresponding CME observed with LASCO. They concluded that structured CMEs can be interpreted as three-dimensional magnetic field entities that arise in a self-similar manner from pre-existing small-scale loop systems. Jing et al. (2004) made a statistical study of more then 100 filament eruptions and found that $56 \%$ of the investigated events corresponded with a CME. Gilbert et al. (2000) performed also a statistical study of prominence activity and developed definitions of active and eruptive prominences. These authors came to the conclusion that eruptive prominences are more strongly associated to CMEs (viz. 94\% of the investigated events) than active prominences (only $46 \%$ ), and that probably all CMEs associated with eruptive prominences possess the three-part front-cavity-core structure. In general, filaments are believed to form in magnetic fields of the shape of sheared arcades and that the prominence material is suspended in the corona by concave upward magnetic fields, possibly possessing some twisted topology (e.g. Kippenhahn and Schlüter, 1957; Kuperus and Raadu, 1974; Manchester, 2001; Karpen et al., 2001; Low and Zhang,

Copyright (c) The Society of Geomagnetism and Earth, Planetary and Space Sciences (SGEPSS); The Seismological Society of Japan; The Volcanological Society of Japan; The Geodetic Society of Japan; The Japanese Society for Planetary Sciences; TERRAPUB.
2004).

Axisymmetric (2.5D) MHD models can be used to numerically simulate CME propagation (e.g. Wu et al., 1999). Chané et al. (2006) demonstrated that 2.5D simulations with a simple CME model, consisting of a high-density plasma blob including a magnetic flux rope, can predict the flow variables at $1 \mathrm{AU}$ for a specific CME event reasonably well. Remark that Jacobs et al. (2007) made a detailed comparison of these 2.5D simulation results to a similar 3D simulation and showed that the 2.5D simulation yields acceptable results provided the momentum of the initial CME is chosen in a proper way. However, it is straightforward to see that the effect of the CME depends on the angular position of the observer and that the assumption of axial symmetry poses severe restrictions to the possible magnetic field configuration in the magnetic cloud. In the present paper, the three-dimensional (3D) extension of this 2.5D model is presented. The strategy followed for simulating a CME event is the same as in Chané et al. (2006), but in stead of launching a spherical plasma blob, a more advanced magnetic flux rope model, with an enhanced density, is flung into the interplanetary medium by giving it an initial velocity profile. The initial magnetic configuration in the flux rope is a modification of the Lundquist equilibrium for constant $\alpha$ force-free fields in cylinder geometry and will be presented in the next section.

\section{Simulation Set-up}

The ideal MHD equations have been solved in spherical coordinates $(r, \theta, \varphi)$ on a three-dimensional spherical mesh, covering a complete sphere, i.e. $\theta \in[0, \pi]$ and $\varphi \in[0,2 \pi]$. The magnetic field is kept divergence-free by using the vector potential on the nodal points. The computational domain covers the region between the lower corona and $30 R_{\odot}$, using a grid resolution of $324 \times 95 \times 184$ cells, including two ghost cells at each boundary. The grid shows 
an accumulation of cells both towards the solar surface and towards the solar equator, where the grid size varies from $\Delta r=0.02 R_{\odot}$ near the solar surface to $\Delta r=0.25 R_{\odot}$ at the outer boundary and from $\Delta \theta=4^{\circ}$ near the poles to $\Delta \theta=0.8^{\circ}$ at the equator. The grid was taken to be equidistant in the azimuthal direction. The simulation was executed on the VIC-cluster of the K.U.Leuven. To reach a time of $t=10 \mathrm{~h}$ in the code, 35382 iterations were needed. The run was finalised in about $21.5 \mathrm{~h}$ by using 120 processors simultaneously.

In order to construct a background solar wind model, the full set of (ideal) MHD equations has been solved in a corotating frame along with an extra added gravitational force as well as an additional heating/cooling source term, very similar to the term that was used by Groth et al. (2000). This wind model shows no dependence on the azimuthal direction and provides a good approximation for the roughly axisymmetric wind occurring at solar minimum.

Romashets and Vandas (2003) present an expression for the magnetic field in toroidal geometry, reading:

$$
\begin{aligned}
B_{r^{\prime}}= & B_{0} \frac{R_{0}-2 r^{\prime} \cos \theta^{\prime}}{2 \alpha R_{0}\left(R_{0}+r^{\prime} \cos \theta^{\prime}\right)} J_{0}\left(\alpha r^{\prime}\right) \sin \theta^{\prime}, \\
B_{\varphi^{\prime}}= & B_{0}\left(1-\frac{r^{\prime}}{2 R_{0}} \cos \theta^{\prime}\right) J_{0}\left(\alpha r^{\prime}\right), \\
B_{\theta^{\prime}}= & B_{0} \frac{R_{0}-2 r^{\prime} \cos \theta^{\prime}}{2 \alpha R_{0}\left(R_{0}+r^{\prime} \cos \theta^{\prime}\right)} J_{0}\left(\alpha r^{\prime}\right) \cos \theta^{\prime} \\
& -B_{0}\left(1-\frac{r^{\prime}}{2 R_{0}} \cos \theta^{\prime}\right) J_{1}\left(\alpha r^{\prime}\right),
\end{aligned}
$$

where $\left(r^{\prime}, \varphi^{\prime}, \theta^{\prime}\right)$ represent the toroidally curved cylindrical coordinates, $J_{0}$ and $J_{1}$ are Bessel functions of the first kind (of order 0 and 1 , respectively), and $R_{0}$ denotes the major axis of the torus. The minor axis is indicated by $r^{\prime}=r_{0}$ and the constant $\alpha$ is determined by $J_{0}\left(\alpha r_{0}\right)=0$. In this way, the magnetic field lines are confined within the torus. This solution for the magnetic field satisfies the solenoidal constraint and approximates the force-free condition in the limit of a large aspect ratio, i.e. in the limit $R_{0} / r_{0} \gg 1$. Next, the Romashets and Vandas solution is subjected to the transformation $r \longrightarrow r-a$ (Gibson and Low, 1998), stretching space inward, towards the origin. The transformation deforms the shape of the original flux rope, resembling an already rising prominence. The stretch factor $a$ is set to a value of $0.3 R_{\odot}$ and the top of the flux rope is at a height of $0.7 R_{\odot}$ above the solar surface. The transformed flux rope solution is then superposed on top of the stationary background wind model discussed above. To keep the analogy with previous work (e.g. Jacobs et al., 2005; Chané et al., 2006) some extra density and velocity is added to the flux rope. The additional density and velocity profiles are both dependent on the toroidal coordinates $r^{\prime}$ and $\varphi^{\prime}$. In doing so, we have control over the added amount of mass, energy, and momentum, and as such over the propagation of the CME.

Prominences are always observed above photospheric regions where the magnetic field changes sign. Since in the applied (axisymmetric) background coronal model for solar minimum the only polarity inversion line coincides with the equator, the flux rope solution is placed above the so-

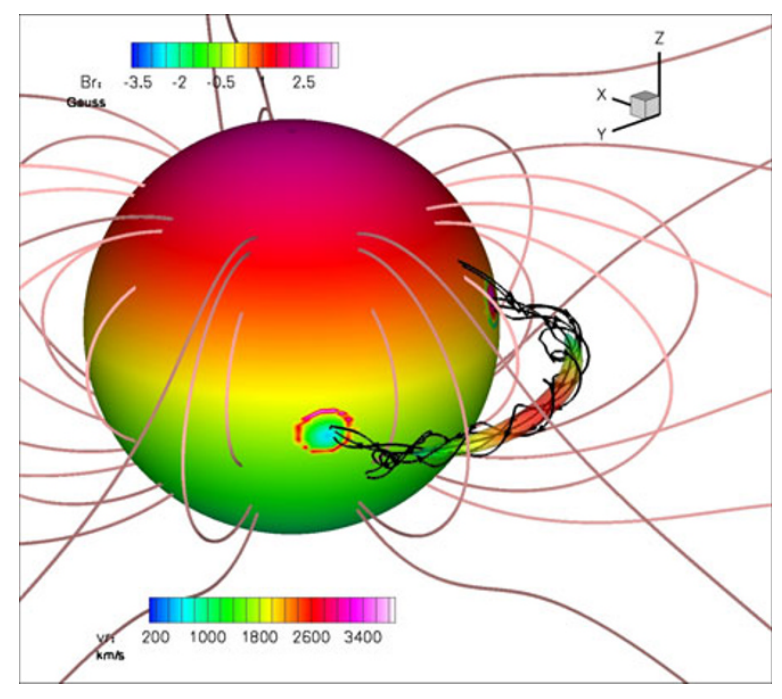

Fig. 1. Initial magnetic field configuration. The solar surface is coloured with the radial magnetic field strength. Inside the flux rope the isosurface of $\rho=2 \rho_{*}$ is plotted, with $\rho_{*}$ the surface density. The isosurface is coloured with the radial velocity.

lar equator in the present simulation. From observations it is known that filaments lie in a highly sheared fashion over the inversion line, making an angle of $\sim 20^{\circ}$ (Leroy et al., 1984). Also according to Leroy et al. (1984), most quiescent prominences are of inverse polarity. The initial configuration of the flux rope was taken such that the observational constraints were satisfied. The magnetic field strength in a quiescent prominence is typically between 5 and 40 Gauss. In the model, the value for the toroidal field in the centre of the flux rope was set to $B_{0}=1.44$ Gauss. This is lower than what is observed, but remember that the initial condition represents a prominence that is already erupting. The maximum velocity inside the flux rope was set to $v_{\text {cme }}=4000 \mathrm{~km} \mathrm{~s}^{-1}$ and the total amount of mass added equals $4 \times 10^{15} \mathrm{~g}$. A visualisation of the initial state is shown in Fig. 1.

\section{Results}

Figure 2 shows contours of the radial velocity at $t=$ 15 min after the onset of the CME event. The initially highly twisted magnetic field in the flux rope reconnects with the overlying magnetic field, but the magnetic field lines in the CME remain connected to the solar surface.

Figure 3 illustrates the longitudinal variation in the density and the velocity. This plot shows the density and radial velocity in the cross-section of three meridional planes and the equatorial plane. The three meridional planes are located at a constant azimuthal position of $\varphi=150^{\circ}$, $\varphi=165^{\circ}$, and $\varphi=180^{\circ}$. The velocity and density profile in the original undisturbed background solar wind are indicated on the plots with at dashed line. From these plots it is clear that a strong shock is propagating ahead of the CME. Since the CME is launched along the negative $X$ axis, the part of the CME in the plane $\varphi=180^{\circ}$ moves the fastest.

Gopalswamy et al. (2005) studied the arrival times of several historical fast events and these authors argue that 


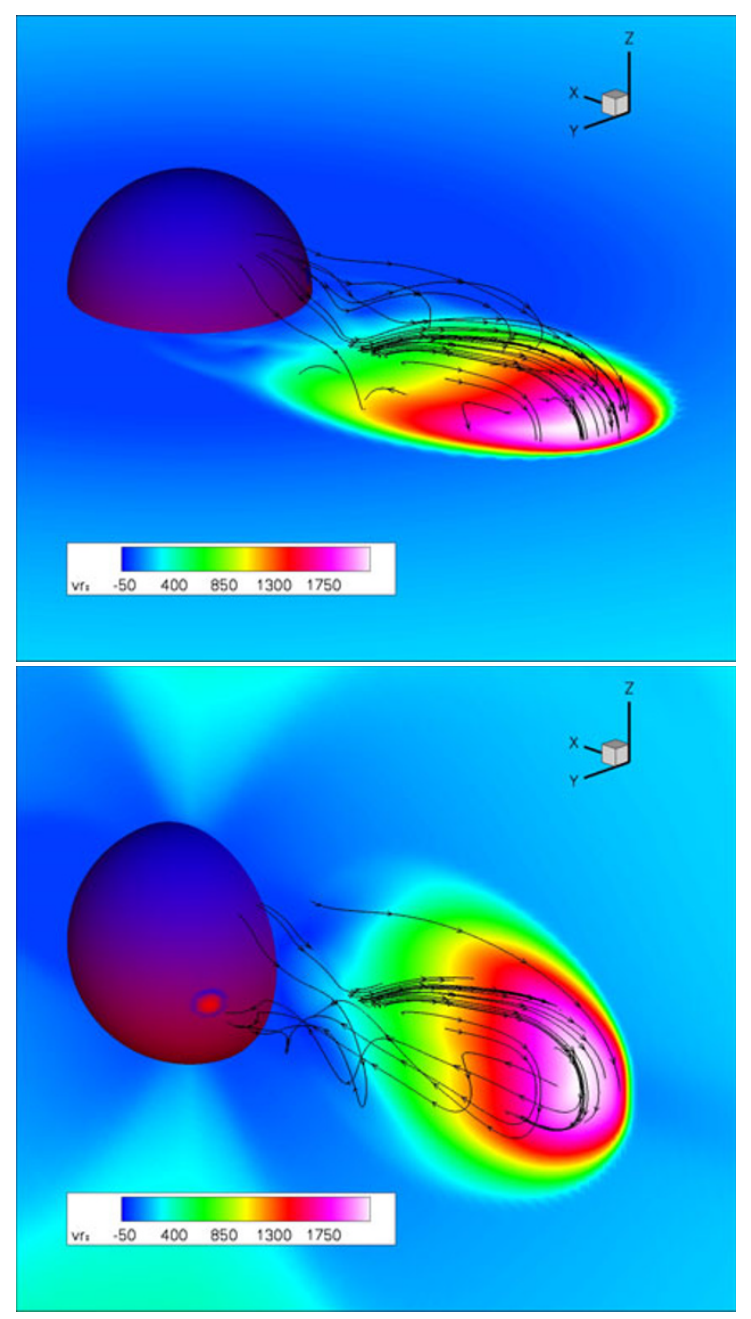

Fig. 2. Snapshot at $t=15 \mathrm{~min}$ showing the radial velocity contours (colour) and the magnetic field lines (black). The solar surface is coloured with the radial magnetic field strength. Top: velocity contours in the equatorial plane; bottom: velocity contours in the $x z$-plane.

the maximum initial speed of a CME may not be much higher than $\sim 3000 \mathrm{~km} \mathrm{~s}^{-1}$. In the present simulation, the plasma blob was given an initial speed in order to mimic the eruption. Since no initiation mechanism was considered, also the mechanism for accelerating CMEs is not captured well in this simulation and the plasma cloud will experience a strong deceleration in the initial phase of the simulation. Therefore, the $v_{\text {cme }}$ parameter is set to a quite high velocity of $4000 \mathrm{~km} \mathrm{~s}^{-1}$. However, remark that this high velocity is only reached in one single point of the flux rope and the average amount of extra velocity added corresponds to a value of only $\sim 600 \mathrm{~km} \mathrm{~s}^{-1}$. The deceleration of the CME is made clear in Fig. 4, where the position of the CME front in the equatorial plane is plotted versus time. The figure shows the height-time plots for the CME front in the three meridional planes discussed above. The plot shows also the height-time profile for the centre of mass, being defines as

$$
\mathbf{r}_{\mathrm{CRM}}=\frac{\int_{V} \mathbf{r} \bar{\rho}(\mathbf{r}) d^{3} \mathbf{r}}{\int_{V} \bar{\rho}(\mathbf{r}) d^{3} \mathbf{r}}
$$
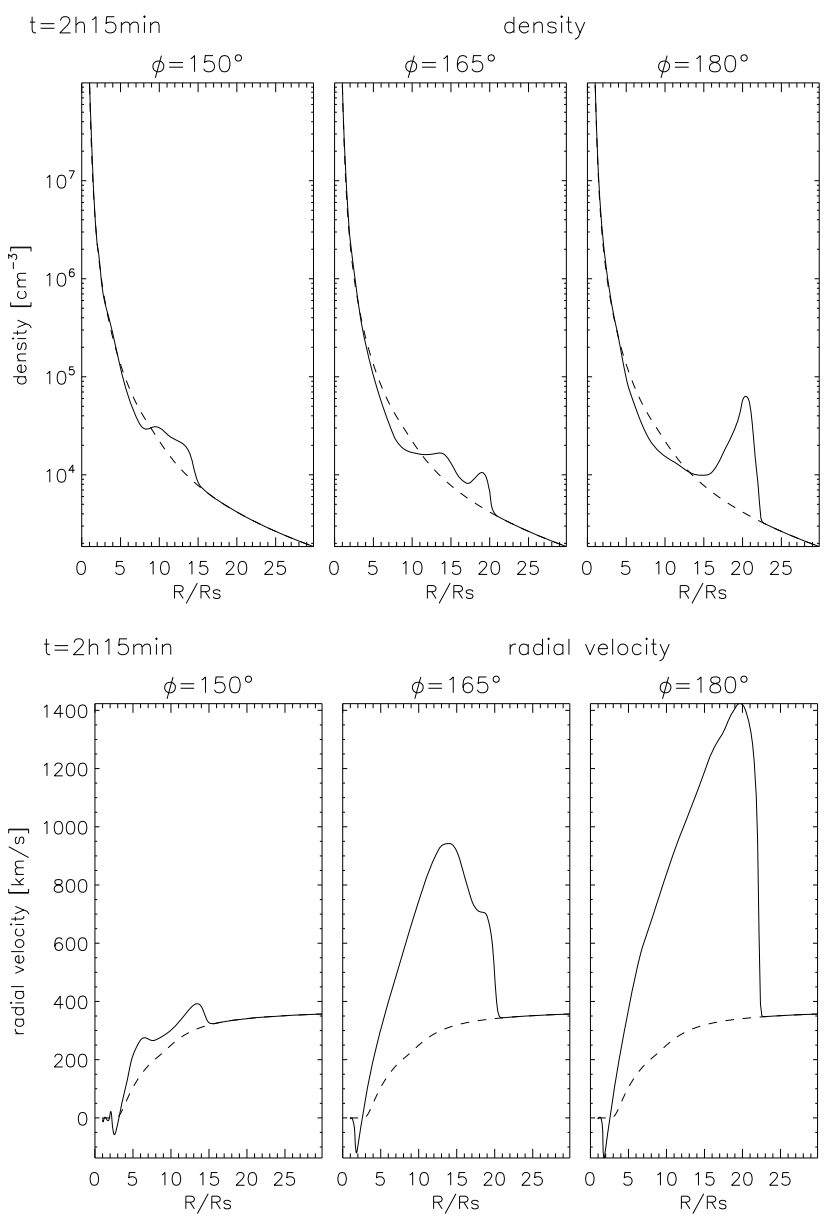

Fig. 3. Cut along the equator of the radial velocity (top) and the density (bottom) at three azimuthal positions, $2 \mathrm{~h} 15 \mathrm{~min}$ after the launch of the $\mathrm{CME}$. The dashed line indicates the steady state wind value.

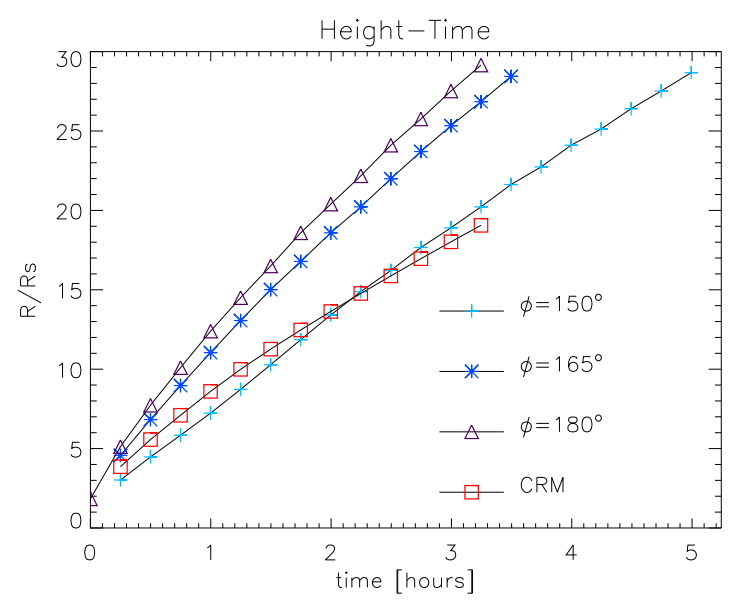

Fig. 4. Height-time curves for the position of the CME front along the equator in three meridional planes and for the centre of relative mass (CRM).

The centre of mass is calculated by using the relative density $\bar{\rho}$, which is a measure for the excess or depletion of the density with respect to the background solar wind density. Since a CME is defined as an outward motion of a new, discrete, bright, white light feature in the coronagraph field of view, only those locations with an enhancement in density 
Table 1. Average velocity and acceleration of the CME front and of the centre of relative mass (CRM).

\begin{tabular}{c|ccc}
\hline & $\varphi=150^{\circ}$ & $\varphi=165^{\circ}$ & $\varphi=180^{\circ}$ \\
\hline$\langle v\rangle$ & $1059 \mathrm{~km} \mathrm{~s}^{-1}$ & $1411 \mathrm{~km} \mathrm{~s}^{-1}$ & $1594 \mathrm{~km} \mathrm{~s}^{-1}$ \\
$\langle a\rangle$ & $-21.3 \mathrm{~m} \mathrm{~s}^{-2}$ & $-48.5 \mathrm{~m} \mathrm{~s}^{-2}$ & $-78.8 \mathrm{~m} \mathrm{~s}^{-2}$ \\
\hline
\end{tabular}

of at least $10 \%$ with respect to the background density were taken into account in the calculation of the centre of mass.

The height-time curves were fitted with first and second order polynomials to obtain the average velocity and acceleration. The measured quantities for the average velocity and acceleration are summarised in Table 1. The CME front (in the $\varphi=180^{\circ}$-plane) shows a strong deceleration of $79 \mathrm{~m} \mathrm{~s}^{-2}$. The extensive statistical study of Yashiro et al. (2004) pointed out that, on average, the acceleration of CMEs with average velocity $\langle v\rangle \geq 900 \mathrm{~km} \mathrm{~s}^{-1}$ is $-15 \mathrm{~m} \mathrm{~s}^{-2}$. The strong deceleration of $79 \mathrm{~m} \mathrm{~s}^{-2}$ of the CME front is rare, but not un-occurring.

\section{Conclusions}

A three-dimensional time dependent MHD simulation of a CME event has been performed, where the simulation domain ranged from the lower corona up to $30 R_{\odot}$. The CME was mimicked by launching a high density flux rope in the solar wind, where the used model for the background wind represents solar minimum conditions. The magnetic field topology of the flux rope was the Romashets and Vandas (2003) solution in toroidal geometry. By applying the transformations of Gibson and Low (1998), the flux rope was stretched towards the solar surface, imitating a rising prominence. The simulation presented in this paper is the $3 \mathrm{D}$ generalisation of the $2.5 \mathrm{D}$ models described in earlier work (e.g. Jacobs et al., 2005; Chané et al., 2006). Threedimensional CME simulations provide the ability to investigate the three-dimensional nature of CMEs. With the recent launch of the STEREO mission, it will become possible to check the outcome of the models with the multiple view point observations made by STEREO.

The model presented here is different from the simulations of e.g. Roussev et al. (2003) and Manchester et al. (2004) in the sense that we add an extra amount of momentum and energy to the flux rope, in order to have more control over its propagation. Roussev et al. (2003) used the flux rope model of Titov and Démoulin (1999) and by removing the overlying line current, the flux rope became unstable, causing an eruption. In case no extra density or velocity is added, the magnetic field of the flux rope in our model simply reconnects with the overlying coronal field, not causing a violent eruption. This might be due to the low magnetic field strength inside the flux rope and a higher magnetic field strength might be desirable. Another weakness of the simulation is the background wind model. The medium in which the CME is propagating influences the evolution of the CME. A more realistic wind model with input from magnetograms and/or empirical laws (e.g. Wang and Sheeley, 1990) for the solar wind is then also necessary if the model wants to be used to simulate specific CME events. For future studies it might be interesting to investigate the effect of the initial parameters like the amount of added ve- locity, density, and magnetic field on the CME evolution.

Acknowledgments. These results were obtained in the framework of the projects GOA/2004/01 (K.U.Leuven), G.0304.07 (FWO-Vlaanderen) and C 90203 (ESA Prodex 8). Financial support by the European Commission through the SOLAIRE Network (MTRN-CT-2006-035484) is gratefully acknowledged. The numerical results were obtained on the HPC cluster VIC of the K.U.Leuven.

\section{References}

Chané, E., B. van der Holst, C. Jacobs, S. Poedts, and D. Kimpe, Inverse and normal coronal mass ejections: evolution up to $1 \mathrm{AU}, A \& A, \mathbf{4 4 7}$, 727-733, 2006.

Cremades, $\mathrm{H}$. and V. Bothmer, On the three-dimensional configuration of coronal mass ejections, $A \& A, \mathbf{4 2 2}, 307-322,2004$.

Gibson, S. E. and B. C. Low, A time-dependent three-dimensional magnetohydrodynamic model of the coronal mass ejection, ApJ, 493, 460-473, 1998.

Gilbert, H. R., T. E. Holzer, J. T. Burkepile, and A. J. Hundhausen, Active and eruptive prominences and their relationship to coronal mass ejections, ApJ, 537, 503-515, 2000.

Gopalswamy, N., S. Yashiro, Y. Liu, G. Michalek, A. Vourlidas, M. L. Kaiser, and R. A. Howard, Coronal mass ejections and other extreme characteristics of the 2003 October-November solar eruptions, J. Geophys. Res., 110, A09S15, 2005.

Groth, C. P. T., D. L. De Zeeuw, T. I. Gombosi, and K. G. Powell, Global three-dimensional MHD simulation of a space weather event: CME formation, interplanetary propagation, and interaction with the magnetosphere, J. Geophys. Res., 105, 25053-25078, 2000.

Jacobs, C., S. Poedts, B. van der Holst, and E. Chané, On the effect of the background wind on the evolution of interplanetary shocks, $A \& A, \mathbf{4 3 0}$, 1099-1107, 2005.

Jacobs, C., B. van der Holst, and S. Poedts, Comparison between 2.5D and 3D simulations of coronal mass ejections, A\&A, 470, 359-365, 2007.

Jing, J., V. B. Yurchyshyn, G. Yang, Y. Xu, and H. Wang, On the relation between filament eruptions, flares, and coronal mass ejections, ApJ, 614, 1054-1062, 2004.

Karpen, J. T., S. K. Antiochos, M. Hohensee, and J. A. Klimchuk, Are magnetic dips necessary for prominence formation?, ApJ, 553, L85L88, 2001.

Kippenhahn, R. and A. Schlüter, Eine Theorie der solaren Filamente, Z. Astrophys., 43, 36-62, 1957.

Kuperus, M. and M. A. Raadu, The support of prominences formed in neutral sheets, $A \& A$, 31, 189-193, 1974.

Leroy, J. L., V. Bommier, and S. Sahal-Bréchot, New data on the magnetic structure of quiescent prominences, $A \& A, \mathbf{1 3 1}, 33-44,1984$.

Low, B. C. and M. Zhang, Magnetostatic structures of the solar corona III. Normal and inverse quiescent prominences, ApJ, 609, 1098-1111, 2004.

Manchester, W., The role of nonlinear Alfvén waves in shear formation during solar magnetic flux emergence, ApJ, 547, 503-519, 2001.

Manchester, W. B., T. Gombosi, I. Roussev, D. L. De Zeeuw, I. V. Sokolov, K. G. Powell, G. Tóth, and M. Opher, Three-dimensional MHD simulation of a flux rope driven CME, J. Geophys. Res., 109, A01102, 2004.

Romashets, E. P. and M. Vandas, Interplanetary magnetic clouds of toroidal shapes, Proc. ISCS 2003 Symposium, 535-540, 2003.

Roussev, I. I., T. G. Forbes, T. I. Gombosi, I. V. Sokolov, D. L. DeZeeuw, and J. Birn, A three-dimensional flux rope model for coronal mass ejections based on a loss of equilibrium, ApJ, 588, L45-L48, 2003.

Titov, V. S. and P. Démoulin, Basic topology of twisted magnetic configurations in solar flares, $A \& A, \mathbf{3 5 1}, 707-720,1999$.

Wang, Y.-M. and N. R. Sheeley, Solar wind speed and coronal flux-tube expansion, ApJ, 355, 726-732, 1990.

Wu, S. T., W. P. Guo, D. J. Michels, and L. F. Burlaga, MHD description of the dynamical relationships between a flux rope, streamer, coronal mass ejection, and magnetic cloud: An analysis of the January 1997 Sun-Earth connection event, J. Geophys. Res., 104, 14789, 1999.

Yashiro, S., N. Gopalswamy, G. Michalek, O. C. St. Cyr, S. P. Plunkett, N. B. Rich, and R. A. Howard, A catalog of white light coronal mass ejections observed by the SOHO spacecraft, J. Geophys. Res., 109, A07105, 2004.

S. Poedts (e-mail: Stefaan.Poedts@wis.kuleuven.be), C. Jacobs, B. van der Holst, E. Chané, and R. Keppens 